\section{The application of human anterior lens capsule autotrans- plantation in phacotrabeculectomy: a prospective, comparative and randomized clinical study}

Conclusions Anterior lens capsule autotransplantation could get comparable outcome as MMC in phacotrabeculectomy with few complications.

Eye (2009) 23, 195-201; doi:10.1038/eye.2008.96; published online 11 April 2008

Keywords: anterior lens capsule; autotransplantation; phacotrabeculectomy; glaucoma

\section{Introduction}

Glaucoma is a severe, potentially blinding disease that affects all age groups and all populations. The aetiology of glaucomatous damage is not very clear yet. Controlling the intraocular pressure (IOP) at an early stage in glaucoma has been shown to slow down or stop progression of the disease. ${ }^{1,2}$ Presently, lowering of IOP is the main goal of glaucoma therapy. Trabeculectomy, introduced by Cairns ${ }^{3}$ in 1968, has become the gold standard filtering procedure for many eyes with glaucoma. However, in some cases, it cannot achieve a good filtering effect because of post-operative fibrosis at the filtering site, which reduce or abolish aqueous flow from the eye into the subconjunctival space. In order to reduce the fibrosis response, antimetabolites such as mitomycin-C (MMC) and 5-fluorouracil (5-FU) have been used in trabeculectomy. However, their antimetabolism effect is often compromised by a number of serious
Tianjin Medical University Eye Center, Tianjin, China

Correspondence: J Ji, Tianjin Medical University Eye Center, 64 Tong'an Road, Heping District, Tianjin, 300070, China

Tel: + 8622 23540698;

Fax: + 862223346434

E-mail: tjykzx@

yahoo.com.cn

Received: 9 July 2007 Accepted in revised form: 28 February 2008 Published online: 11 April 2008 
ocular complications, such as bleb leakage, prolonged hypotony, epitheliopathy, blebitis, endophthalmitis, etc. ${ }^{4-8}$

Biomaterials have been studied to enhance the success of filtering procedures, such as horse hair, silk threads, acrylic plates, gelatin, amniotic membrane, etc. They are too expensive for most patients in the developing countries to use, and these materials are not autogenous. Anterior lens capsule (ALC), the thickest basement membrane of our body, ${ }^{9}$ is a constitutive basement membrane of the lens. Use of ALC graft for chronic corneal ulcers or as support material in RPE cell transplantation has been well described in literature. ${ }^{9-12}$ ALC transplantation has been shown to be effective in resolution of these problems. It does not result in fibrosis or rejection reaction, and components of humoral immunity do not participate in immune reaction. ${ }^{13}$

The presence of ALC itself or some cytokine released may keep fibroblasts from forming adhesion. We think that the prevention of fibroblast stimulation is beneficial for maintaining a functional bleb after filtration surgery. Therefore, we utilized ALC in trabeculectomy for patients with cataract and glaucoma to prevent postoperative adhesion. To the best of our knowledge, there are few studies in literature evaluating the efficacy of ALC autotransplantation in glaucoma surgery.

\section{Materials and methods}

\section{Patients}

This study was approved by the ethics committee of Tianjin Medical University Eye Center, Tianjin, China. Fifty consecutive Chinese patients (Table 1) with coexisting cataract and glaucoma were enrolled from Tianjin Medical University Eye Center and divided into two groups randomly using the random number table. Written, informed consent was obtained from all patients. The inclusion criteria were visually significant cataract plus uncontrolled glaucoma with an IOP over $21 \mathrm{~mm} \mathrm{Hg}$ despite maximum tolerable medical therapy. The patients with history of uveitis, previous ocular surgery, or systemic diseases, such as diabetes mellitus, were excluded from our study. No subjects refused to enrol and that none were lost to follow-up during the course of the study.

All the patients underwent a thorough ocular examination, including record of best corrected visual acuity (BCVA) and slit-lamp biomicroscopy. All IOP recordings (Goldmann applanation tonometry) were made between 09:00 and 12:00. BCVA preoperatively ranged from $20 / 500$ to 'hand movements'.
Table 1 Baseline patient characteristics for ALC and MMC groups

\begin{tabular}{llll}
\hline Characteristics & ALC group & MMC group & P-value \\
\hline Sex & & & \\
$\quad$ Male & $13(44.8 \%)$ & $9(42.9 \%)$ & 0.89 \\
$\begin{array}{l}\text { Female } \\
\text { Mean age in }\end{array}$ & $16(55.2 \%)$ & $12(57.1 \%)$ & \\
$\begin{array}{l}\text { years (SD) } \\
\text { Surgical }\end{array}$ & ALC implant & MMC & 0.75 \\
procedure & & & \\
Diagnosis & & & \\
$\begin{array}{l}\text { POAG } \\
\text { AACG }\end{array}$ & 2 & 1 & \\
CACG & 17 & 8 & \\
Pre-operative & $23.1 \pm 8.2(9.3-38)$ & $23.4 \pm 7.7(10.2-36)$ & 0.9 \\
IOP (mm Hg) & & & \\
Numbers of & $2.5 \pm 1.1(1-5)$ & $2.4 \pm 1.1(1-5)$ & \\
medications & & & \\
\hline
\end{tabular}

Abbreviations: AACG, acute angle closure glaucoma; ALC implant, anterior lens capsule implant; CACG, chronic angle closure glaucoma; IOP, intraocular pressure; MMC, mitomycin-C trabeculectomy; POAG, primary open angle glaucoma.

\section{Preparation of human anterior lens capsule}

Human ALC was obtained under sterile condition from the patient when they received continuous curvilinear capsulorhexis. Scrape the lens epithelial cells off ALC carefully with a lenticular hook under the operation microscope until ALC was completely denuded. Wash ALC twice with a sterile, balanced salt solution before use.

\section{Surgical technique}

All the surgeries were performed by the same experienced surgeon (JJ), and followed by an ophthalmologist (WL) who was masked to the type of surgery performed. The pupil was dilated with $0.5 \%$ tropicamide drops (Shuanghe Co. Ltd, Beijing, China) 30 min before surgery.

We performed one-site phacotrabeculectomy. All the surgeries were made under peribulbar local anaesthesia with 2\% lidocaine (Jinyao Co. Ltd, Tianjin, China). Ocular compression was performed in every case. A fornixbased conjunctival flap was fashioned at the 11 o'clock position.

In the ALC group, the conjunctival flap was followed by a $2 / 3$ thickness sclero-corneal tunnel, which was prepared by dissecting the sclera forward into the clear cornea with a crescent knife. A small limbal paracentesis was performed at the $2 o^{\prime}$ clock position. The anterior chamber was then entered along the scleral tunnel with the 3.2-mm keratome. The chamber was immediately 
deepened with Viscoat (SA Alcon-Couvreur NV, Rijksweg, Puurs, Belgium). Pupil stretching or synechiolysis were performed when necessary. After the continuous curvilinear capsulorhexis, the nucleus was removed with a standard four-quadrant, 'divide and conquer' technique. An automated irrigation/aspiration apparatus (Alcon Laboratories Inc., Fort Worth, TX, USA) was introduced into the anterior chamber to remove the cortical remnants and to polish the posterior lens capsule. The intraocular lens (IOL) was placed in the capsular bag. The pupil was contracted with $0.01 \%$ carbachol injection (Bausch \& Lomb Freda, Jinan, Shandong, China). A trabeculectomy of $1 \times 2 \mathrm{~mm}$ was performed under the same sclero-corneal tunnel with a $15^{\circ}$ scalpel and Vannas scissors. A peripheral iridectomy was performed, which was basally large enough to be sure that iris could not be visualized in the base of the trabeculectomy opening. ALC was then placed under the scleral tunnel and kept it as flat as possible. The scleral tunnel was closed with one 10/0 nylon suture, meanwhile, ALC was sutured in the scleral tunnel. The conjunctival wound was closed with $8 / 0$ vicryl continuous sutures. No antimetabolites were used during or after surgery.

In the MMC group, a sponge soaked with $0.2 \mathrm{mg} / \mathrm{ml}$ MMC was applied under the conjunctival flap for $2 \mathrm{~min}$, and the area was washed out with a balanced salt solution. Phacoemulsification and trabeculectomy were then done in the same way as in the ALC group. ALC was not used in this group.

\section{Post-operative care and follow-up}

Post-operative medications employed routinely are $0.3 \%$ ofloxacin drops (Santen, Osaka, Japan) six times per day, $1 \%$ fluorometholone drops (Santen) six times per day, and $0.1 \%$ diclofenac sodium drops (Xingqi

Pharmaceutical Co. Ltd, Shenyang, Liaoning, China) four times per day. All the drops were stopped after 6 weeks of treatment, and steroids were tapered off during the 6 weeks. Subconjunctival steroids, post-operative suturelysis or 5-FU injections were not used in each group.

Post-operative evaluation was conducted on days 1, 7 and months 1, 3, 6, and 12. The patients, who needed closer follow-up, according to the clinical outcome, were evaluated monthly or even weekly. On each occasion, the patients were fully examined under the slit lamp biomicroscopy. The BCVA, IOP, and bleb appearance were determined. Any complications of surgery were noted. Status of the ALC grafts was detected with the ultrasound biomicroscopy (UBM; Paradigm Medical Industries, Salt Lake City, UT, USA) 1 month after surgery.
For the purpose of the study, the surgery was considered a 'complete success' when IOP was $\leqslant 21 \mathrm{~mm} \mathrm{Hg}$ without antiglaucoma medication, a 'qualified success' when IOP was $\leqslant 21 \mathrm{~mm} \mathrm{Hg}$ with antiglaucoma medication. If patients had an IOP $>21 \mathrm{~mm} \mathrm{Hg}$ regardless of medications or had progressive glaucomatous atrophy with visual field loss, they were classed as surgical failures.

The filtering bleb was divided into four types: (1) polycystic functioning bleb, (2) diffuse functioning bleb, (3) flat nonfunctioning bleb, and (4) encapsulated nonfunctioning bleb. ${ }^{14}$

\section{Statistical analysis}

Data was reported as mean $\pm \mathrm{SD}$. All analysis was done with SPSS software version 13.0. $\chi^{2}$-Test was used for the number of filtering bleb, the success of the surgery, and BCVA; $t$-test was used for the pre-operative and post-operative comparisons of IOP. The number of antiglaucoma medications was analysed by employing a rank sum test. $P<0.05$ was considered statistically significant. The success of the technique was analysed using a Kaplan-Meier cumulative survival curve.

We certify that all applicable institutional and governmental regulations concerning the ethical use of human volunteers were followed during this research.

\section{Results}

\section{Appearance of filtering bleb}

After the 12 months' follow-up, in the ALC group, type I and type II bleb were seen in 5 and 19 eyes, respectively, that is to say, the functional bleb accounted for $82.8 \%$ $(24 / 29)$, type III bleb were seen in 5 eyes; in the MMC group, type I and type II bleb were seen in 2 and 17 eyes, respectively, the functional bleb accounted for $90.5 \%$ $(19 / 21)$, type III bleb was noted in 2 eyes. No type IV bleb was found in both groups. There was no statistical significant difference between the two groups in the rate of functional bleb ( $\chi^{2}$ test, $\chi^{2}=0.132, P=0.716$ ).

\section{Success of the technique and numbers of antiglaucoma medications}

Kaplan-Meier survival analysis representing longitudinal complete success rates is shown in Figure 1.

In the ALC group, 28 eyes (96.6\%) were considered 'complete success' and 1 eye 'qualified success' that needed one additional antiglaucoma medication 1 month postoperatively. Two eyes were 'qualified success' that needed one medication, 1 eye fulfilled the criteria for 'failure' 3 months postoperatively. Four eyes were 
'qualified success', 1 eye was 'failure' 6 months postoperatively, and thereafter, the success rate was $82.8 \%$.

In the MMC group, all the eyes (100\%) were considered 'complete success' 1 month postoperatively. One eye was 'qualified success' that needed one medication 3 months postoperatively. Two eyes were 'qualified success' 6 months postoperatively and thereafter, the success rate was $90.5 \%$.

We also obtained good post-operative IOP control with a significant reduction of antiglaucoma medications. In the ALC group, the mean number of antiglaucoma medications reduced from 2.5 preoperatively to 0.2 at the last post-operative visit. In the MMC group, the mean number of antiglaucoma medications reduced from 2.4 preoperatively to 0.1 at the last post-operative visit. There was no statistical significant difference between the two groups in the number of antiglaucoma medications before and after surgery (Mann-Whitney's $U$-test, $Z=-0.435,-0.800, P=0.663,0.424$, respectively).

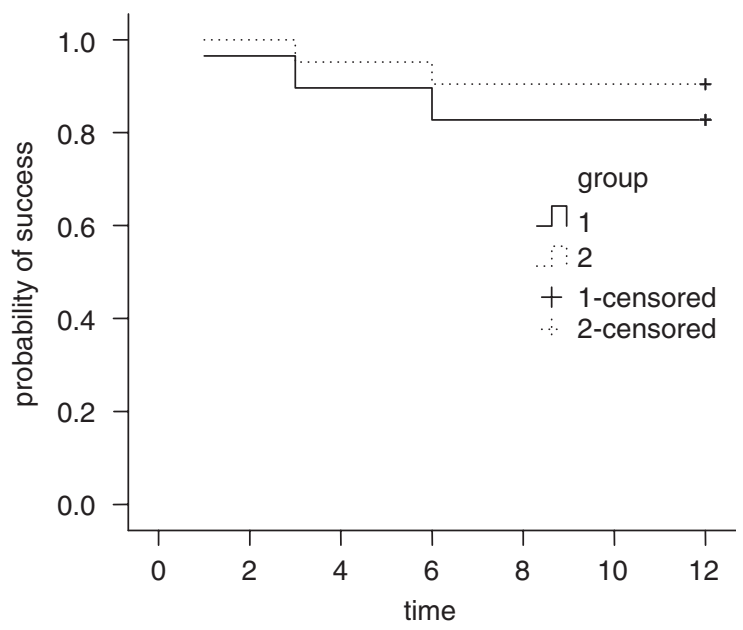

Figure 1 Kaplan-Meier cumulative probability of successful glaucoma control (intraocular pressure $\leqslant 21 \mathrm{~mm} \mathrm{Hg}$ without antiglaucoma medication) over 1 year. Group1 is the anterior lens capsule (ALC) group; the success rate was 96.6, 89.7, 82.8\% at months 1, 3, 6 and thereafter. Group2 is the mitomycin-C (MMC) group; the success rate was 100, 95.2, 90.5\% at months 1 , 3,6 and thereafter.

\section{IOP change}

Comparison of the pre-operative and end of follow-up indices yielded significant improvement in IOP. In the ALC group, the IOP decreased from $23.1 \pm 8.2 \mathrm{~mm} \mathrm{Hg}$ before surgery to $13.5 \pm 3.4 \mathrm{~mm} \mathrm{Hg}$ at the final examination (paired samples $t$-test, $t=7.022, P=0.000$ ). In the MMC group, the IOP decreased from $23.4 \pm 7.7 \mathrm{~mm} \mathrm{Hg}$ before surgery to $13.5 \pm 2.9 \mathrm{~mm} \mathrm{Hg}$ after (paired samples $t$-test, $t=7.059, P=0.000$ ). The percentages of IOP decrease were $35 \%$ in the ALC group and $37 \%$ in the MMC group, and there was no statistical significant difference between them (independent samples $t$-test, $t=-0.370, P=0.713$ ). Comparison of IOP at each time point in both groups is shown in Table 2 .

\section{BCVA change}

At 12 months postoperatively, 12 eyes in the ALC group had BCVA $>20 / 30,8$ eyes in the MMC group had $B C V A>20 / 30$, there was no statistical significant difference between them $\left(\chi^{2}=0.055, P=0.815\right)$.

\section{$U B M$}

All the ALC graft could be detected by UBM 1 month after surgery (Figure 2). The high-level echo under the scleral tunnel represented the ALC graft, the low-level echo under the conjunctiva represented the filtering bleb.

\section{Complications}

There were no intra-operative complications noted.

In the ALC group, five eyes had shallow anterior chamber and one had significant choroidal detachment. Corneal oedema and post-operative iridocyclitis were mild. In each case these complications settled spontaneously in one week without the need for surgical intervention. No patients suffered wound leakage, endophthalmitis or rejection reaction.

In the MMC group, four eyes had shallow anterior chamber and two had significant choroidal detachment; one eye had wound leakage that pressure dressing and

Table 2 IOPs following operation $(\mathrm{mm} \mathrm{Hg})($ mean $\pm \mathrm{SD})$

\begin{tabular}{llllllll}
\hline & Pre-operative & 1 day & 1 week & 1 month & 3 months & 6 months & 1 year \\
\hline ALC group & $23.1 \pm 8.2$ & $9.2 \pm 5.1$ & $8.8 \pm 3.0$ & $12.6 \pm 4.0$ & $12.7 \pm 4.0$ & $13.4 \pm 3.5$ & $13.5 \pm 3.4$ \\
MMC group & $23.4 \pm 7.7$ & $7.3 \pm 4.0$ & $7.7 \pm 1.9$ & $11.9 \pm 3.6$ & $12.2 \pm 2.9$ & $13.1 \pm 2.8$ & $13.5 \pm 2.9$ \\
$t$ & -0.129 & 1.453 & 1.456 & 0.651 & 0.517 & 0.342 & 0.007 \\
$P$ & 0.898 & 0.153 & 0.152 & 0.518 & 0.608 & 0.734 & 0.994 \\
\hline
\end{tabular}

Abbreviations: ALC implant, anterior lens capsule implant; MMC, mitomycin-C.

The IOP decreased significantly after surgery in both groups and there was no statistical significant difference between the two groups at each time point. 


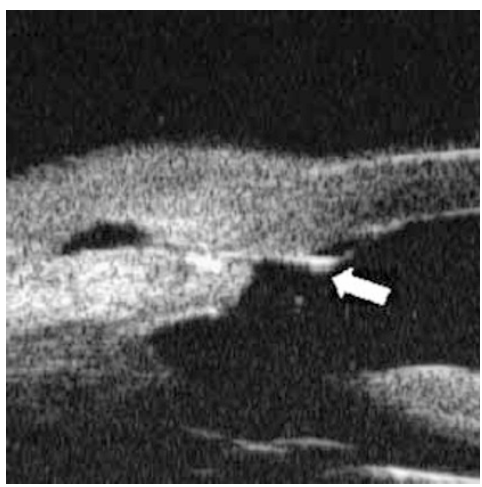

Figure 2 The anterior lens capsule (ALC) graft could be found in the filtering site 1 month after surgery (white arrow).

mydriasis could not help, so we had to suture the conjunctiva to cure the leakage.

\section{Discussion}

ALC is the production of lens epithelial cells, it is an acellular, soft, smooth, transparent membrane that maintains the shape of the lens. It comes from the ectoderm and is composed of 30-40 layers of lamella whose thickness is $30-40 \mathrm{~nm}$. Central ALC thickness is $8.2 \pm 5.5 \mu \mathrm{m}$ optically and $12.4 \pm 2.5 \mu \mathrm{m}$ histologically. ${ }^{15}$

Basement membrane collagen from ALC type IV collagen and peptides from the noncollagenous domain of the $\alpha 3$ (IV) chain, corresponding to residues 185-203 and 179-208, inhibit tumour cell proliferation, specifically through the interaction of the -SNStripeptide (residues 189-191) with the CD47/ $\alpha \mathrm{v} \beta 3$ integrin receptor complex. The $\alpha 3$ (IV)185-203 and the $\alpha 3(\mathrm{IV}) 179-208$ peptides, designated as oncothanin, regulate endothelial cell (EC) proliferation, adhesion, and motility that ultimately inhibit angiogenesis. The $\alpha 3$ (IV) chain biologically active peptides bind specifically to the integrin $\alpha v \beta 3$ and its accessory receptor CD47, which regulate a number of functions in the cell. Integrin $\alpha v \beta 3$ reactive monoclonal antibodies have been shown to inhibit tumour formation and growth by inhibiting angiogenesis. The CD47 reactive monoclonal antibody alone can inhibit angiogenesis thus suggesting a potential role in the regulation of angiogenesis. ${ }^{16,17}$ The antiangiogenic activity of collagen IV also has been reported by Maeshima et al, ${ }^{18,19}$ who demonstrated that peptides derived from the $\alpha 3$ chain, specifically the 3 (IV)69-88 and $\alpha 3$ (IV)74-98, inhibited EC proliferation and angiogenesis by promoting EC apoptosis through binding to the $\alpha v \beta 3$ integrin in an RGD-independent manner.

Besides its antiangiogenesis effect, there are other contributions of ALC transplantation in our procedure:
ALC aids filtration by the capillary action; ALC is an avascular basement membrane, it keeps the sclerotomy site mechanically open by preventing adhesions of the scleral flap and the scleral bed; what's more, ALC derives from the ectoderm and the sclera from the mesoderm, tissues from different blastoderms do not adhere with each other; ${ }^{20}$ ALC is autologous, it contains no immunogenic living cells, and it will not lead to rejection reaction. ${ }^{13}$

The wound healing process after filtration surgery is a series of complicated biological reactions, which include sludged blood formation period, proliferation period, granuloma formation period, and collagen formation period. In patients without high risk factor for failure, the migration and proliferation of fibroblasts postoperatively culminate in 3-5 days and terminate in 11 days. So it is of great importance to interfere in the reactions within 2 weeks after surgery. ${ }^{21-23}$ In our research, all the ALC grafts could be found by UBM 1 month postoperatively, thus ensuring an effective antiscarring function.

The complications noted after our procedure, such as shallow anterior chamber, corneal oedema, and iridocyclitis are similar to conventional phacotrabeculectomy, and can not be incriminated to ALC autotransplantation.

Previous studies using MMC have reported a number of serious late ocular complications as a direct consequence of the use of this agent. Depending on the concentration and exposure time, an incidence of hypotony ranging between 3 and $32.7 \%$ has been reported. ${ }^{5,6,24}$ In our series, ocular hypotony developed in none of the 29 eyes. No patient in our series suffered hypotony related maculopathy - a complication that has previously been reported in $3-17.3 \%$ of patients receiving MMC. ${ }^{5,6,24}$ Late bleb infection after the use of antimetabolites is a major concern, and it is remarkable that no cases of bleb infection or endophthalmitis occurred in our series. Wolner et al, ${ }^{25}$ with a mean followup of 23.7 (16.3) months, reported an incidence of bleb related endophthalmitis of 5.7\% (13/229) after 5-FU trabeculectomy and Higginbotham et al, ${ }^{8}$ with a mean follow-up of 18.5 (10.8) months, reported an incidence of $2.6 \%(6 / 229)$ after MMC trabeculectomy. Our study finds little risk of vision loss from hypotonous maculopathy and suggests that the intermediate term risk of bleb-related infection and endophthalmitis may be less than that reported with the use of antimetabolites.

Although a randomized prospective study comparing ALC autotransplantation with placebo would be preferable, such a study would be difficult to justify in the light of previous studies, which show a low success rate without antimetabolites treatment in these circumstances. 
Cataract and glaucoma are common conditions that occur frequently in the same patient. Historically, the approach to the patient with coexisting visually significant cataract and glaucoma has not been uniform. Following isolated cataract surgery, IOP increase in the early post-operative period is more severe in eyes with glaucoma. In addition, subsequent cataract surgery performed in eyes with functioning filtering bleb is associated with a bleb failure rate of up to $30 \%$. On account of these problems, combined filtration and cataract surgery is more attractive when indicated. ${ }^{26} \mathrm{We}$ consider that another major factor in the high rate of cataract surgery accompanying filtering surgery in this series is the introduction of phacoemulsification in our unit. Phacotrabeculectomy is associated with a lower incidence of post-operative complications and achieves better control of astigmatism and earlier visual rehabilitation than glaucoma surgery and ECCE. ${ }^{27}$ This has lowered our threshold for intervention in such cases. The one-site combined surgery employed in this series is also faster and easier to perform than two-site combined surgery, because it does not require a scleral flap, the surgeon can use the sclero-corneal tunnel instead.

Our results of ALC autotransplantation in phacotrabeculectomy suggest that good mid-term control of IOP can be achieved without the need for antimetabolites. It is considerable in patients who may not be able to comply with a complex medical regimen, particularly in the developing countries where the patients' compliance is poor and antimetabolites may not be available. ALC is available in every combined surgery, obviating the need to obtain additional materials and reducing costs for the patients. Majority of the patients enjoyed good visual rehabilitation and successful pressure reduction 1 year after the surgery. The absence of significant complications related to the surgery is also encouraging. It is our belief that more widespread application of combined surgery may be appropriate.

\section{References}

1 Mao LK, Stewart WC, Shields MB. Correlation between intraocular pressure control and progressive glaucomatous damage in primary open-angle glaucoma. Am J Ophthalmol 1991; 111: 51-55.

2 Chauhan BC, Drance SM. The relationship between intraocular pressure and visual field progression in glaucoma. Graefes Arch Clin Exp Ophthalmol 1992; 230: 521-526.

3 Cairns JE. Trabeculectomy: preliminary report of a method. Am J Ophthalmol 1968; 66: 673-679.

4 Bindlish R, Condon GP, Schlosser JD, D'Antonio J, Lauer KB, Lehrer R. Efficacy and safety of mitomycin-C in primary trabeculectomy. Ophthalmology 2002; 109: 1336-1341.
5 Shields MB, Scroggs MW, Sloop CM, Simmons RB. Clinical and histopathologic observations concerning hypotony after trabeculectomy with adjunctive mitomycin $\mathrm{C}$.

Am J Ophthalmol 1993; 116: 673-683.

6 Zacharia PT, Depperman SR, Schuman JS. Ocular hypotony after trabeculectomy with mitomycin C. Am J Ophthalmol 1993; 116: 314-326.

7 Greenfield DS, Suner IJ, Miller MP, Kangas TA, Palmberg PF, Flynn Jr HW. Endophthalmitis after filtering surgery with mitomycin. Arch Ophthalmol 1996; 114: 943-949.

8 Higginbotham EJ, Stevens RK, Musch DC, Karp KO, Lichter $\mathrm{PR}$, Bergstrom TJ et al. Bleb related endophthalmitis after trabeculectomy with mitomycin C. Ophthalmology 1996; 103: 650-656.

9 Kozak I, Trbolova A, Zibrin M, Komorova T, Kolodzyeiski L, Juhas T. Electron microscopic study of anterior lens capsule allotransplants in chronic corneal ulcers. Cornea 2004; 23: 797-803.

10 Lee CJ, Vroom JA, Fishman HA, Bent SF. Determination of human lens capsule permeability and its feasibility as a replacement for Bruch's membrane. Biomaterials 2006; 27: 1670-1678.

11 Kiilgaard JF, Wiencke AK, Scherfig E, Prause JU, La Cour M. Transplantation of allogenic anterior lens capsule to the subretinal space in pigs. Acta Ophthalmol Scand 2002; 80: 76-81.

12 Hartmann U, Sistani F, Steinhorst UH. Human and porcine anterior lens capsule as support for growing and grafting retinal pigment epithelium and iris pigment epithelium. Graefes Arch Clin Exp Ophthalmol 1999; 237: 940-945.

13 Kozak I, Trbolova A, Rosocha J, Jautova J, Juhas T, Ledeckv V. Immunofluorescent analysis of anterior lens capsule allotransplantation for chronic corneal ulcers. Cesk Slov Oftalmol 2004; 60: 261-266.

14 Kanski JJ. The glaucomas. In: Kanski JJ (ed). Clinical Ophthalmology. Butterworth-Heinemann: Oxford, 1999, pp 183-262.

15 Ziebarth NM, Manns F, Uhlhorn SR, Venkatraman AS, Parel JM. Noncontact optical measurement of lens capsule thickness in human, monkey, and rabbit postmortem eyes. Invest Ophthalmol Vis Sci 2005; 46: 1690-1697.

16 Brooks PC, Montgomery AM, Rosenfeld M, Reisfeld RA, $\mathrm{Hu}$ T, Klier G. Integrin alpha v beta 3 antagonists promote tumor regression by inducing apoptosis of angiogenic blood vessels. Cell 1994; 79: 1157-1164.

17 Shahan TA, Grant DS, Tootell M, Ziaie Z, Ohno N, Mousa S et al. Oncothanin, a peptide from the alpha3 chain of type IV collagen, modifies endothelial cell function and inhibits angiogenesis. Connect Tissue Res 2004; 45: 151-163.

18 Maeshima Y, Manfredi M, Reimer C, Holthaus KA, Hopfer $\mathrm{H}$, Chandamuri BR et al. Identification of the antiangiogenic site within vascular basement membranederived tumstatin. J Biol Chem 2001; 276: 15240-15248.

19 Maeshima Y, Yerramalla UL, Dhanabal M, Holthaus KA, Barbashov S, Kharbanda $S$ et al. Extracellular matrixderived peptide binds to $\alpha v \beta 3$ and inhibits angiogenesis. J Biol Chem 2001; 276: 31959-31968.

20 Anwar M, el-Sayyad F, el-Maghraby A. Lens capsule inclusion in trabeculectomy with cataract extraction. J Cataract Refract Surg 1997; 23: 1103-1108.

21 Chang L, Crowston JG, Gordeiro MF, Akbar AN, Khaw PT. The role of the immune system in conjunctival wound healing after glaucoma surgery. Surv Ophthalmol 2000; 45: $49-68$. 
22 Cordeiro MF, Chang L, Lim KS, Daniels JT, Pleass RD, Siriwardenad D et al. Modulating conjunctival wound healing. Eye 2000; 14: 536-547.

23 Khaw PT, Chang L, Wong TTL, Maed A, Daniels JT, Cordeiro MF. Modulation of wound healing after glaucoma surgery. Curr Opin Ophthalmol 2001; 12: 143-148.

24 Mégavand GS, Salmon JF, Scholtz RP, Murray AD. The effect of reducing the exposure time of mitomycin $\mathrm{C}$ in glaucoma filtering surgery. Ophthalmology 1995; 102: 84-90.

25 Wolner B, Liebmann JM, Sassani JW, Ritch R, Speaker M, Marmor M. Late bleb-relatedend ophthalmitis after trabeculectomy with adjunctive 5-fluorouracil. Ophthalmology 1991; 98: 1053-1060.

26 Singh RP, Goldberg I, Mohsin M. The efficacy and safety of intraoperative and/or postoperative 5-fluorouracil in trabeculectomy and phacotrabeculectomy. Clin Exp Ophthalmol 2001; 29: 296-302.

27 Cagini C, Murdolo P, Gallai R. Longterm results of one-site phacotrabeculectomy. Acta Ophthalmol Scand 2003; 81: 233-236. 